\title{
CDISC SDTM Severity Intensity Scale for Adverse Event Terminology
}

National Cancer Institute

\section{Source}

National Cancer Institute. CDISC SDTM Severity Intensity Scale for Adverse Event

Terminology. NCI Thesaurus. Code C66769.

Terminology codelist used with Severity Intensity Scale for Adverse Event within the Clinical Data Interchange Standards Consortium Study Data Tabulation Model. 